\title{
Architecture of e-Learning
}

\author{
Mafawez Alharbi and Mahdi Jemmali
}

\begin{abstract}
Recent years, traditional learning has moved to electronic learning (e-learning). Many institutions and university has forced on e-learning in. In the form of e-learning, instructors and learners live in different places and they do not engage in a classroom environment, but within virtual space. There are small number of e-learning architecture have proposed in the literature. However, the proposed architecture has lack of embedding intelligent system in the architecture of e-learning. In this paper, we propose an architecture and algorithm for the problem of e-learning. This research has proposed architecture for e-learning. Thus there is intelligence built into the architecture and thus the e-learning system is automatically responsive to the user requirements.
\end{abstract}

Index Terms - PLE, VLE, algorithm, architecture, e-learning, software engineering.

\section{INTRODUCTION}

E-learning has increased in recent years; many institutions have invested in e-learning system to develop their employee. In the form of e-learning, instructors and learners live in different places and they do not engage in a classroom environment, but within virtual space. Instructors and learners do not meet face to face, but they meet in a virtual space [1]. E-learning is defined in [2] as technology-based learning in which learning materials are delivered electronically to remote learners via computer. Using E-learning system can get many advantages such as Student and learners have ability to take their courses anytime anywhere. In addition, E-learning system is able to help disable people who have special need to study from their home.

There is few proposed architecture for e-learning system in the literature. However, the current architecture for e-learning has some limitations. One of these limitations is that it not designed to be intelligent system. Therefore, to the best of our knowledge, there is no architecture for e-learning that can be described intelligent system.

This research has proposed architecture for e-learning. Thus there is intelligence built into the architecture and the e-learning system is automatically responsive to the user requirements. The e-learning architecture is automatically responsive to each individual user on demand, and it is able to predict user preferences or interests. In addition, this research has focused in identifying an algorithm for one of the component for e-learning architecture.

The rest of this paper is organized as follow: an

Manuscript received September 9, 2015; revised November 27, 2015 This work was supported in part by the Majmaah University, Saudi Arabia.

Mafawez Alharbi is with the College of Science, Majmaah University, Saudi Arabia (e-mail: m.alharbi@mu.edu.sa).

Mahdi Jemmali was with the College of Science, Majmaah University, Saudi Arabia. He is also with MARS Research Unit, Monastir University (e-mail: m.jemmali@mu.edu.sa). introduction to e-learning system will be discus in Section II. Proposed of e-learning architecture with its details will be presented in Section III. In Section IV, Stages of the proposed system will be presented. Functionality of Intelligent component will be discus in Section V.

\section{E-LEARNING}

\section{A. Introduction to e-Learning}

There are two types of education based on either traditional or electronic learning (e-learning).The way of delivering education has been changed recently, traditional learning environments have moved to electronic education (e-learning). E-learning is rapidly developing area especially in education. It [3] suggests that e-learning are tools to support lifelong learning. E-learning is presented as a solution to lifelong learning

E-learning has increased in recent years; many institutions have invested in e-learning system to develop their employee. In the form of e-learning, instructors and learners live in different places and they do not engage in a classroom environment, but within virtual space. When instructors and learners do not meet face to face, and they meet in a virtual space, this is called "e-learning".

The technology for e-learning has been widely accepted by many institutions, university and companies. There was a dream for learning to be available any time anywhere. This dram today is real, and this is because the increasing the research for creating new architecture for e-learning [4].

There are many definitions for e-learning in the literature. One of these definitions for E-learning it's a technology-based learning in which learning materials are delivered electronically to remote learners via computer. This would decrease face to face contact between teacher and learner. In addition, there is more attention take place offline. Higher education is moving now to use technology in order to deliver online courses and decrease traditional learning [5].

\section{B. Advantages of e-Learning}

E-learning has many advantages; these advantages have been presented in [6]-[8] and reviewed below:

The number of the students go to the high education are increasing every years. Using e-learning will have good benefits for theses student because they can view their modules online. In addition, e-learning will help universities to reduce the number of physical space.

Student and learners has ability to take their courses anytime anywhere. In contrast to traditional learning where learner restricted in physical place and time.

E-learning system is able to help disable people who have special need to study from their home. In addition, people who have family commitment, e-learning is so important for them [9]. 
Organization can get many benefit for using e-learning, this will contribute to reducing the overall cost. In addition, the work will be deliver seamlessly this is because the broadband would be ubiquitous.

\section{Related Work}

The concept of e-learning is developed recently. The complexity of designing e-learning architecture system has become obscurity which is being address by research. Current e-learning platforms are developed using existing technologies and compensate for the disadvantages of traditional education methods. Existing architecture of e-learning has used technology and raise from the limitations of the traditional learning [10]. There are small numbers of e-learning architecture are presented in the literature.

First e-learning architecture is presented by Wilson on 2005 [11]. Wilson called its architecture "the future of VLE". This architecture is high level architecture; the updated version for the proposal is presented in [12]. It is an alternative design pattern for educational systems that emphasizes symmetric connections with a range of services both in formal and informal learning, work, and leisure, and identifies strategies for implementation and experimentation. The most serious limitation in this architecture is that this is no intelligent component in the architecture and this is no details in this architecture [13].

Another proposal is presented in [14]. The author is introduced a multi-agent approach for design architecture of e-learning. The proposed approached composes a system into a set of stages, where each stage is about a multi-agent system composed of a number of agents. When an agent cannot complete a duty, it asks other agents to collaborate in achieving that duty. The limitation in this proposal is that presenting architecture of e-learning is focus on the content of the curriculum and on transform Knowles and information [15].

\section{Limitations of e-Learning}

In the previous part, some architecture has been presented. It shows that building architecture for e-learning is challenging task.

However, these proposed architectures have some limitations. The users of these architectures are receiving too much information. To the bet of our knowledge, this is no architecture for e-learning that has ability to filter e-resources. In more details, the current architecture of e-learning is not intelligent enough. The user of this architecture is not receiving its need. In addition, the current e-learning architecture has lack of restricted feature; the contents are restricted for a particular time and place. Our proposal approach will be presented in the next part [16].

\section{Proposed of E-LEARning ARchiteCture}

This research has proposed architecture for e-learning. Thus there is intelligence built into the architecture and thus the e-learning system is automatically responsive to the user requirements. The e-learning architecture is automatically responsive to each individual user on demand, and it is able to predict user preferences or interests. In addition, this research also has focused in identifying an algorithm for one of the important component for e-learning architecture.

The proposed architecture is presented on Fig. 1 and consists of the following components: User Interface, User profile, E-provider, Acquisition, Filtering, History database, Creator, Evaluator, Good rank, Bad rank and Selector.

More details on our approach are presented below:

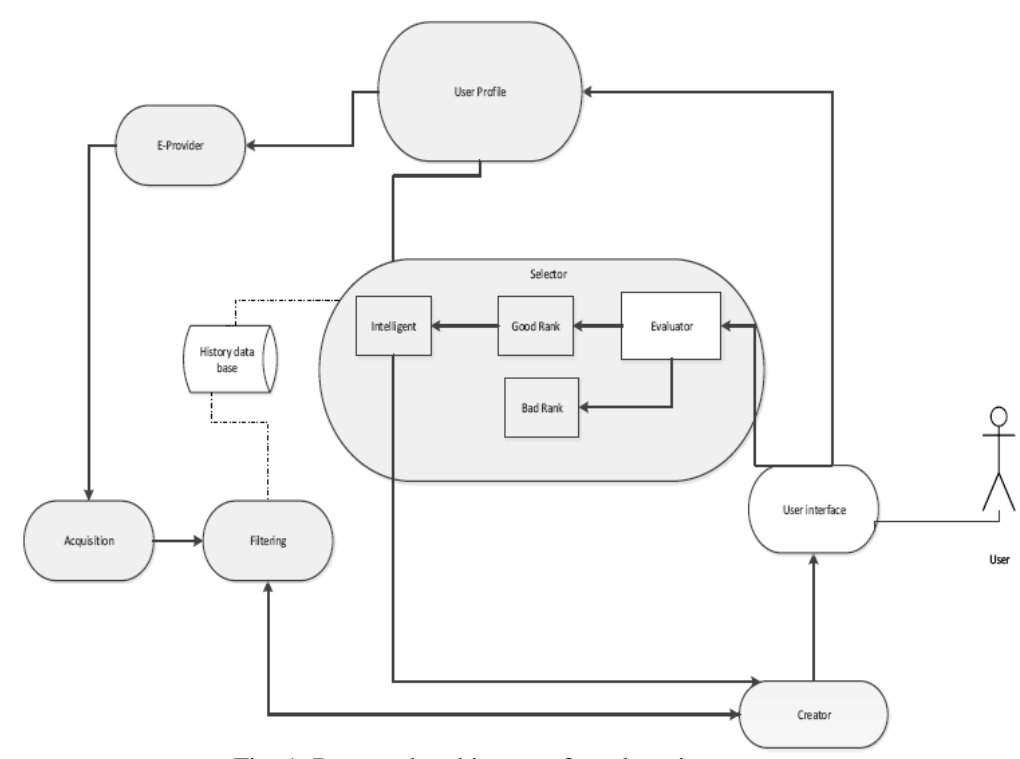

Fig. 1. Proposed architecture for e-learning system.

\section{A. User Interface}

The user of the system is able to interact with the system through this component. The user interface is an application which can be deployed on PC, laptop or smart mobile device. The user of this system will be able to manage its profile by the user interface. In addition, the user has ability to give its feedback for what has been received from the system through this component. The system will be able to alert the user to any activity which is so important to the user. In contrast, the user interface will be used to communicate with the user.

\section{B. User Profile}

The user of the system is able to provide information regarding their interest. The user profile can change from time to time and the user can modifies its profile directly. 
User profile can be contains basic information for the user such as user name, age, mobile number, e-mail and address. The information in this component is changed rarely. User can database that contains links to the e-resources which the user are interested in. this databases are organized by the user.

\section{E-Provider}

E-Provider has ability to gather e-resources from different sources and to supply Acquisition component with appropriate e-recourse. For example E-provider can be IEEE Explore. In addition, the user of the system is able to define its E-provider in its user profile.

\section{Acquisition}

The function of the Acquisition component is that it is able to isolate e-resources from e-provider and forward it to the Filtering component. This component has Application Program Interface (API), in order to interact with different E-providers.

\section{E. Filtering Component}

This component uses a technique that has ability to filter all the e-resource and provider only a new e-resources for the user. Any e-resources which the user has read before the system will stop processing it. This component is an essential because it has ability to prevent duplication of e-resources which has been sent to the user in early stage.

\section{F. History Data Base}

This data base contains all the e-recourse, which the user of the system has received. Any e-resources that have been sent to the user must be storage in this database. This database has a communication with filtering component.

\section{G. Creator}

The objective of creator component is to create an action (message) in order to send it to the user. The message should be adapt to the user's mobile devices. The component receives a message from Filtering and AI components.

\section{H. Selector}

Selector component has ability to analysis user feedback and make some knowledge after more evaluation by the user. Selector component consists of four subsystems which are: Evaluator, good rank, bad rank and intelligent component. All these components are explained below:

\section{1) Evaluator}

The Evaluator component has the ability to receive a feedback from the user based on what has been read. This component has some mechanism, which has ability to analysis the feedback for the user and identifies a good and bad e-resource. The result from this component consists of two part good or bad e-resources.

\section{2) Good rank}

This component has storage the highest e-resources which are ranked by the user as good e-resources. The result from Good Rank component will feed up the intelligent component, in order to predicate e-resources that might need by the user.

\section{3) Bad rank}

This component storage the lowest rank for e-resources, which are ranked by the user as bad e-resources.it can be consider that bad e-resources by all resources which have bad evaluation by the user. This evaluation can be considered by application of simple or complex evaluation.

\section{4) Intelligent component}

The intelligent component is able make some knowledge after more evaluation has been done by the user. This is based on the information received from three components (Good rank, user profile and History database). This component is able to learn from the habit from the user. The result from this component should be delivered to the Creator component.

The AI component will have an apprenticeship after application of several iterative searches. It can send an auto evaluation (score) of e-resource to user to help them to make decision for the e-resource. This auto evaluation based in history modeling and history evaluation make by the user. The AI will be explained in more details in part 5 .

\section{STAGES OF THE SYSTEM}

Each system has stages to follow, in order to complete the task. In this part we will present these stages for the proposed system. These stages are shown as steps which show how our proposed architecture can work. These steps are presented in Fig. 2 and it consists of nine steps as follow:

Step 1:

The user of proposed architecture has ability to interact with the system via the user interface component. This is the only way for the user to interact with the proposed system.

Step 2:

The user of the system is able to provide the proposed system with any information relating to her various interests. The user profile component allow the user to update (create, edit and delete) its profile via the user interface.

Step 3:

Once the user has completed information, the user can ask the system to start it duty by clicking on search option, which is on the user interface component. The search request will take the information from the user profile and send this information to the e-provider.

Step 4:

The main object for e-provider is to gather e-resource from different place. E-provider is able to search for e-resources and send the result to the Acquisition component.

Step 5:

Acquisition component has ability to isolate e-resources form the e-provider and forward it to the Filtering component.

Step 6:

The main function for the Filtering component is to prevent duplication of the e-resources. This means that, this component has ability to provide only a new e-resource for the user. If the e-recourse is new for the user, then the system will continue processing this e-resource. Assuming that the e-recourse is new for the user, then a request must be sent to the Creator component. Otherwise, the e-resources will be stop to process.

Step 7:

The creator component is able to create an action based on what has been received from the Filtering and AI component and then send it to the user via the user interface. 


\section{Step 8:}

The user of the proposed system can give its feedback based on what has been received. Selector component is responsible for receiving the feedback from the user. This main that once the user has view and read the e-resources.
User can give its feedback based on the e-resources which has been received.

Step 9:

Intelligent component has ability to predicate some knowledge after more evaluation by the user.

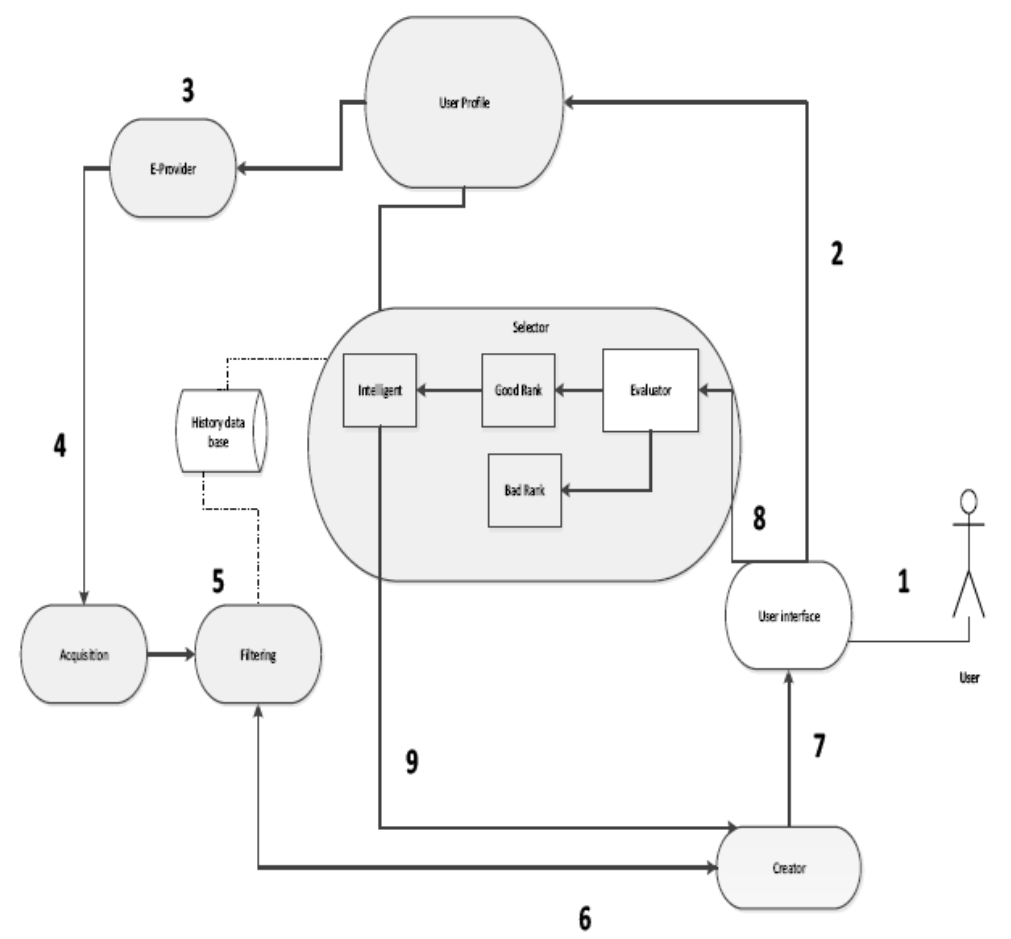

Fig. 2. System architecture stages.

\section{FunCTIONALITY OF INTELLIGENT COMPONENT}

There are two man functions for intelligent components. The first one is that it has ability to predicate e-recourse for the user. The intelligent component is able to predicate e-recourse for the user, based on the information received from three components (Good rank, user profile and information history). This component is able to learn from the feedback from the user. The result from this component should be delivered to the Creator component. For implementing the intelligent component Artificial Intelligent (AI) techniques must be used.

The second function is to make some knowledge after more evaluation by the user. We turn our attention to the description of an effective evaluation which constitutes the second key feature of our study. This evaluation is in the spirit of the approach that has been implemented by modeling and iterative strategy. Basically, the proposed model is a multi-start local search method which requires iteratively solving a sequence of multi instances. The intelligent component can make some knowledge after more evaluation by the user. This component will have an apprenticeship after application of several iterative searches. It can send an auto evaluation (score) of e-resource to user to help him to make decision for the e-resource. This auto evaluation based in history modeling and history evaluation make by user.

\section{A. Description Model}

After an iterative searching of different e-resources we can make model based on the following items.

\section{1) Notations}

Domain: Certainly, the user make search an e-resource based to one domain of work or research. We denote the domain by $d$.

Subject: All users make search starting from a subject. So, we can always fixed subject of our research. We denote the subject by $s$.

Keywords: We denote the keywords by $k$.

References: We denote the references by $r$.

Kind of e-resource: We denote the Kind of e-resource by $k i$.

Number of pages: We denote the number of pages by $n b$.

Authors: We denote the authors by $A u$.

Provider: We denote the provider by $P$.

Now we have 8 variables: $d, s, k, r, k i, n b, A u, P$.

The most important variables to consider is: $d, s, k$. Therefore, we can give a highest ponderation of these variables as following:

$d:[0-20]$

$s:[0-20]$

$k:[0-15]$

The remained variables have the following ponderations:

ki: [0-10]

$n b:[0-10]$

Au: [0-10]

$P:$ [0-5]

We denote:

$$
D^{1}=\operatorname{Max}(d+s+k) \text { and } D^{2}=\operatorname{Max}(k i+n b+A u+P)
$$

The optimal case is evaluated where we find our search. In this case, $D^{1}=55$ and $D^{2}=45$. We denoted by $D_{\max }^{1}$ the value 
of $D^{1}$ and $D_{\max }{ }^{2}$ the value of $\mathrm{D}^{2}$ in this optimal case. Now we describe the score as following:

$$
\text { Score }=d+s+k+r+k i+n b+Q u+P
$$

If score $>D_{\max }{ }^{1}$ then it consider a good score. Else, it is consider as a bad score. It's clearly; the optimal case has the maximum value of score which equal to 100 . We denoted by $D_{\text {max }}$ this maximum score.

The score generated by IC can varied from 0 to 100 .

In our architecture we consider the evaluation by user by using rank. The rank giving by user varied between 1 and 5 . We can give the signifying rank as following:

1) Irrelevant

2) Fine

3) of interest

4) relevant

5) very relevant

Now we have to convert the score calculated automatically by the IC to the proposed rank as it shows in Table I.

TABLE I: GENERATE PROPOSED RANK FROM CALCULATED SCORE

\begin{tabular}{cc}
\hline \hline Score & Rank \\
\hline$[0-25]$ & 1 \\
{$[26-54]$} & 2 \\
{$[55-70]$} & 3 \\
{$[71-85]$} & 4 \\
{$[86-100]$} & 5 \\
\hline \hline
\end{tabular}

These rank converted from score will be proposed by IC to user.

The IC will generate the score automatically based on history evaluations by user. We can find that the IC can't deliver score before fixed number of iteration denoted by iter $_{\text {max }}$.

Each iteration represents one search carry out by user. So, each search provides one evaluation by use. After iter $_{\text {max }}$ evaluations the IC can calculate score. Let iter $_{\max }=1000$.

The user can make decision to accept or to modify the proposed rank by IC.

\section{B. Evaluation of IC}

1) Percentage of success

Denote by counter the variable which contained the number of coincidence of proposed rank by IC and the rank chosen by user.

Case 1: Accept proposed rank by IC. In this case, counter will be incremented.

Case 2: User doesn't accept the proposed rank by IC and he chooses another rank.

In fact, the number of acceptances or refuse of the proposed rank can be huge or small. For this raison, we must make evaluation of IC.

Let $C_{p}$ the number of evaluation make by user. So, we can calculate the percentage of coincidence of proposed rank by IC and the rank chosen by user. Denoted by Eval this percentage:

$$
\text { Eval }=\frac{\text { counter }}{C_{p}} \times 100
$$

This percentage represents the success of proposed rank.
We can fix the percentage of satisfaction denoted by $S_{\text {score }}$. So, when Eval is less than $S_{\text {score }}$ the IC must be revised.

In fact, if Eval $\geq 70 \%$ than we can say that "we have a good IC" else we will do some enhancements for IC.

In practice, we can fix a number denoted by $n b_{\max }$ which represent thenumber of evaluations by user necessary to make evaluation to the IC for each time.

\section{2) Enhancement of IC}

Let the functions:

Generate_score(): This function generate automatically score by IC based in history evaluation of user.

Update_compter(): Function which calculate counter

Change_D1() : Function which attribute modification to $D^{1}$

Change_D2(): Function which attribute modification to $D^{2}$

The algorithm is described below:

Multi-start score (MSS)

Step $0 . n b=0$

Step 1. Change_D2()

$n b=n b+1$

Step 2.fori $=1$ to $C_{p}$ do

Generate_score()

$$
\text { Update_compter() }
$$

$$
\text { End for }
$$

Step 3. Eval $=\left((\right.$ compter $\left.) /\left(C_{p}\right)\right) * 100$

Step 4. If Eval $\geq 70 \%$ then exit

else if $n b<$ niter $D_{2}$ goto Step 1

else

Step $5 . n b=0$

Step 6. Change_D1()

$n b=n b+1$

Step 7.fori $=1$ to $C_{p} d o$

Generate_score()

$$
\text { End for }
$$

Update_compter()

Step 8. Eval $=\left((\right.$ compter $\left.) /\left(C_{p}\right)\right) * 100$

Step 9. If Eval $\geq 70 \%$ then exit

else if $n b<$ niterD1 goto Step 6

else exit

\section{End if}

\section{End if}

If we exit the algorithm (MSS) with $n b=$ niterDl then meaning we haven't obtained the $70 \%$ of satisfaction. In this case we must choose the better percentage.

\section{CONCLUSION}

There are small number of e-learning architecture have proposed in the literature. However, the proposed architecture has lack of embedding intelligent system in the architecture of e-learning. In this paper, we propose an architecture and algorithm for the problem of e-learning. This research has proposed architecture that is intelligence built into the architecture and the e-learning system is automatically responsive to the user requirements.

The goal for this work is to propose intelligent architecture for e-learning system. This work has focused on building algorithm for intelligent component which is one of the important components for the architecture. We expect that some of the ideas that have been developed in this paper would prove useful for the e-learning problems, but this 
would require further investigation. Now days, e-learning is still in earlier stage, there are many issues need to investigate more.

\section{ACKNOWLEDGMENT}

We thank anonymous referees for their helpful comments on earlier versions of our paper. This research was supported in part by Majmaah University, Saudi Arabia.

\section{REFERENCES}

[1] E. V. Raaij and J. Schepers, "The acceptance and use of a virtual learning environment in China," Computer Education, vol. 50, pp. 838-852, 2008

[2] J. L. Zhao, L. Zhou, J. F. Nunamaker, and D. S. Zhang, "Can e-learning replace classroom learning?" Communications of the ACM, pp. 75-79, 2004.

[3] G. Attwell, "Personal learning environments-the future of elearning?" E-Learning Papers, vol. 2, pp. 1-8, 2007.

[4] B. Duan, H. M. Hosseini, K. V. Ling, and R. K. L. Gay,"An architecture for online laboratory e-learning system," International Journal of Distance Education Technologies (IJDET), vol. 4, no. 2, pp. 87-101, 2006.

[5] K. Sharma, D. Sood, A. Singh, and P. Pandit, "Strategic architecture for e-learning at HP University," International Journal of Educational Management, vol. 24, no. 7, pp. 575-596, 2010.

[6] C. Y. Y. Cheng and J. Yen, "Virtual learning environment (VLE): A web-based collaborative learning system," presented at Thirty-First Hawaii International Conference, Hawaii, 1998.

[7] S. Stojanov, I. Ganchev, I. Popchev, M. S. O'Droma, and R. Venkov "DeLC-distributed eLearning center," presented at 1st Balkan Conference on Informatics (BCI'2003), Greece, 2003.

[8] V. Waller, "The state of the nation: E-learning in the 21st century," Training Journal Ely, 2004.

[9] N. Sclater, "Web 2.0, personal learning environments, and the future of learning management systems," Research Bulletin, Boulder, vol. 13, CO: EDUCAUSE Center for Applied Research, 2008.

[10] D. Zhou, Z. Zhang, S. Zhong, and P. Xie, "The design of software architecture for e-learning platforms," Technologies for e-Learning and Digital Entertainment, Springer, pp. 32-40, 2008.

[11] S. Wilson, "Future VLE — The visual version," Scott's Workblog, 2005.

[12] S. Wilson, O. Liber, M. Johnson, P. Beauvoir, P. Sharples, and C. Milligan," Personal learning environments: Challenging the dominant design of educational systems," Journal of e-Learning and Knowledge Society, pp. 27-38, 2007.

[13] T. Valtonen, S. Hacklin, P. Dillon, M. Vesisenaho, J. Kukkonen, and A. Hietanen, "Perspectives on personal learning environments held by vocational students," Computers and Education, pp. 732-739, 2012.

[14] S. Hammami, H. Mathkour, and E. A. Al-Mosallam, "A multi-agent architecture for adaptive E-learning systems using a blackboard agent," Computer Science and Information Technology, pp. 184-188, 2009.

[15] A. Al-Ajlan, "Service oriented computing for dynamic virtual learning environments," De Montfort University, 2009.

[16] S. Wilson et al., "Personal learning environments: Challenging the dominant design of educational systems," Interactive Learning Environments, vol. 16, pp. 1-2, 2008.

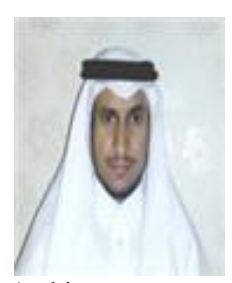

Arabia.

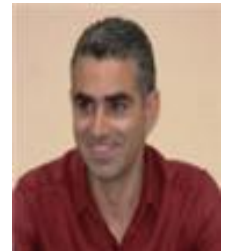

Mafawez Alharbi is an assistant professor of Computer Science Department, College of science, Majmaah University, Saudi Arabia. Currently, he is the vice deanship for IT at Majmaah University. He received his $\mathrm{Ph}$. D. in computer science, De Montfort University, UK, 2014; the MS in software engineering, De Montfort University, 2010, UK; and the BS in computer science, King Saud University, 2004, Saudi

Mahdi Jemmali is an assistant professor of Computer Science Department, College of science, Majmaah University, Saudi Arabia. He is also in membership of Research Unit MARS, Monastir University. He received his Ph.D. in Polytechnic School of Tunisia and Management Higher Institute. His research interests are OR and algorithms. 